ISSN 0103-5150

Fisioter. Mov., Curitiba, v. 25, n. 2, p. 379-388, abr./jun. 2012 Licenciado sob uma Licença Creative Commons

\title{
Associação entre as alterações posturais e a respiração bucal em crianças
}

\author{
Association between postural changes and \\ mouth breathing in children
}

\author{
Tissiani Morimoto ${ }^{[a]}$, Ana Paula Barcellos Karolczak ${ }^{[b]}$ \\ [a] Fisioterapeuta graduada pela Universidade do Vale do Rio dos Sinos (UNISINOS), São Leopoldo, RS - Brasil, e-mail: \\ tissi.morimoto@gmail.com \\ [b] Fisioterapeuta, Mestre em Ciências do Movimento Humano, professora do curso de Fisioterapia da Universidade \\ do Vale do Rio dos Sinos (UNISINOS), Centro Universitário Lasalle (UNILASALLE), Canoas, RS - Brasil, e-mail: \\ anapbk@gmail.com
}

\section{Resumo}

Introdução: Durante o período de crescimento e desenvolvimento corporal, é possível que surjam alterações posturais que podem decorrer de uma respiração incorreta, por via bucal. Objetivos: Verificar se a presença de alterações posturais tem relação com a presença da respiração bucal em crianças de segunda a quarta série do Ensino Fundamental. Materiais e métodos: Participaram do estudo 117 crianças com idade média de 9,017 £ 0,851 anos, sendo 54 meninos e 63 meninas. A avaliação foi constituída por um questionário sobre sinais e sintomas da respiração bucal e uma avaliação postural fotogramétrica com software para Avaliação Postural $\left(\mathrm{SAPO}^{\circledR}\right)$. Para a análise da comparação entre os grupos, foram utilizados os testes t-student e Mann Whitney; e para a análise da associação, o teste Exato de Fisher. Foi considerado p $\leq 0,05$ como nível de significância. Resultados: Em relação ao tipo de respiração, prevaleceu, de forma significativa, a respiração bucal em relação à nasal ( $\mathrm{p}<0,001)$. Quanto ao alinhamento vertical da cabeça, predominou a anteriorização em ambas as vistas. No que diz respeito à assimetria horizontal da escápula, houve predomínio da assimetria à esquerda e, para o alinhamento horizontal da pelve, predominou a anteroversão em ambas as vistas. Na medida do ângulo perna/retropé, prevaleceu o calcâneo valgo direito e esquerdo. As diferenças observadas entre os grupos, respirador bucal e nasal, não se mostraram significativas $(p \geq 0,05)$. 
Conclusão: Foi encontrado um alto percentual de crianças com sinais e sintomas da respiração bucal e alterações posturais, porém sem relação entre os achados.

Palavras-chave: Respiração bucal. Postura. Fisioterapia (Especialidade).

\section{Abstract}

Introduction: During the period of growth and body development, it is possible to occur changes in posture that may result from wrong breathing, through the mouth. Objectives: To determine whether the presence of postural changes is related to the presence of mouth breathing in children from second to fourth grade of elementary school. Materials and methods: The study included 117 children with mean age of $9.017 \pm 0.851$ years, 54 males and 63 females. The evaluation consisted of a questionnaire about signs and symptoms of mouth breathing and a photogrammetric postural assessment with the software $S A P{ }^{\circledR}$. The analysis of the comparison between groups was made by student's t-test and Mann-Whitney; and for association analysis it was used the Fisher Exact test. It was considered $p \leq 0.05$ as significance level. Results: Regarding the type of breathing, prevailed, significantly, mouth breathing compared to nasal breathing $(p<0.001)$. For the vertical alignment of head, anteriorization predominated in both views. For the horizontal asymmetry of scapula, there was a predominance of asymmetry to the left. For the horizontal alignment of the pelvis, anteversion predominated in both views. In measuring the angle leg/hindfoot, prevailed calcaneal valgus right and left. The differences observed between groups of mouth breathing and nasal breathing were not significant ( $p \geq 0.05$ ). Conclusion: It was found a high percentage of children with signs and symptoms of mouth breathing and postural changes, but it was not found relationship between this findings.

Keywords: Mouth breathing. Posture. Physical therapy (Specialty).

\section{Introdução}

Durante o período de crescimento e desenvolvimento corporal, que compreende a infância e adolescência, é possível que surjam alterações posturais (1), as quais podem decorrer, dentre outros motivos, de uma respiração incorreta, por via bucal (2). Alterações posturais são consideradas normais até os oito anos, pois após essa idade, o corpo se adapta e realinha. Porém, quando a criança respira pela boca, essas alterações persistem durante todo período de crescimento e desenvolvimento (3).

A Síndrome do Respirador Bucal, que pode estar relacionada com obstruções nasais, hábitos bucais inadequados ou fatores genéticos, é verificada quando a substituição da respiração nasal por respiração bucal se dá durante um período prolongado (4). Essa síndrome se caracteriza por distúrbios dos órgãos da fala e articulações, associados com alterações funcionais, estruturais, patológicas, posturais, oclusais e comportamentais (2). Alguns estudos (5-7) concordam com as características citadas, ao referirem como reflexos da respiração bucal, aspectos como: insônia, agitação, hiperatividade, dificuldade de aprendizagem, sonolência diurna, cansaço frequente, olheiras, ronco, baba noturna, baixo apetite, crescimento físico diminuído e alterações posturais.

As alterações posturais desencadeadas pela respiração bucal estão relacionadas ao fato de o corpo humano se adaptar para facilitar a passagem de ar das vias aéreas superiores às vias aéreas inferiores, promovendo a anteriorização e a extensão da cabeça (8-10). Como os músculos trabalham de forma sinérgica e são organizados em cadeias, esse deslocamento da posição da cabeça, que muda o centro de gravidade, altera toda a mecânica corporal (8). Dentre as alterações posturais desencadeadas pela respiração bucal, estão, além da anteriorização e extensão da cabeça, a anteriorização dos ombros, o aumento da lordose lombar, a anteroversão pélvica, o abdômen protruso, os joelhos valgos e os pés planos $(8,11-13)$.

Todas as linhas de pesquisa de mecânica corporal consideram que a boa postura pode ser verificada mediante o alinhamento vertical de cinco pontos do corpo: os pontos médios das orelhas, as articulações do ombro, quadril, joelho e tornozelo. 0 alinhamento estático desses pontos é fundamental para o estado dinâmico. No período de crescimento, existe uma flexibilidade do organismo, a qual torna possível que o crânio se adapte em função de exigências mecânicas de uma postura 
ereta. Sendo assim, quando ocorre uma adaptação anatômica do crânio devido a alguma alteração da função fisiológica do sistema respiratório, os músculos das costas e do pescoço ficam responsáveis pelo equilíbrio da cabeça, que se inclina para frente (14).

Santos et al. (15) creditam as consequências prejudiciais à função de sustentação e mobilidade às alterações posturais, tornando muito importante seu diagnóstico precoce para uma intervenção fisioterapêutica eficiente, principalmente em crianças com sistema musculoesquelético complacente. Além disso, caso não haja uma intervenção durante a fase de desenvolvimento, os padrões posturais inadequados podem se tornar permanentes. Breda e Moreira (13) acrescentam que o tratamento deve ser iniciado o mais precocemente possível, destacando a importância da abordagem correta da causa do padrão de respiração alterado, tornando o prognóstico do paciente mais favorável. Esse tratamento deve ser realizado por uma equipe multidisciplinar, com atuação da fisioterapia não só no tratamento das alterações posturais, mas também no padrão respiratório (16).

As alterações posturais presentes na infância são, no entanto, muitas vezes creditadas a fatores externos. Detsh et al. (1) mencionam que as crianças fazem a utilização de mochilas com peso inadequado, sentam de forma incorreta e adotam comportamentos de risco à coluna. Dessa forma, não são comumente investigadas outras causas ou realizadas associações a outras alterações presentes em uma criança respiradora bucal.

Com base no exposto, torna-se relevante uma investigação sobre a presença de sinais e sintomas da respiração bucal em escolares e sua relação com a presença de alterações posturais. Faz-se necessária uma pesquisa para alertar sobre esse problema que, se não tratado precoce e corretamente, poderá acarretar problemas funcionais, físicos e comportamentais.

Sendo assim, o presente estudo tem como objetivo verificar se a presença de alterações posturais tem relação com a presença de sinais e sintomas da respiração bucal em crianças do Ensino Fundamental.

\section{Materiais e métodos}

Identificação do tipo de estudo e população

Esta pesquisa possui uma concepção metodológica de abordagem quantitativa, tratando-se de um estudo observacional, de caráter descritivo (17).
A população deste estudo foi constituída por estudantes de segunda a quarta séries do Ensino Fundamental de duas escolas públicas do Vale do Sinos - RS, com idades entre 8 e 10 anos.

Os critérios de exclusão adotados foram: estudantes que apresentaram o questionário incompleto, ou que apresentaram doenças genéticas e/ou congênitas com envolvimento do sistema musculoesquelético, doenças musculoesqueléticas, neuromusculares ou degenerativas.

Para um coeficiente de correlação de 0,3, nível de significância de $5 \%$ e poder de $90 \%$, a amostra deveria ser composta por 113 estudantes (17). Inicialmente, foram avaliados 120 estudantes; entretanto, dois apresentaram doenças neuromusculares e um apresentou questionário incompleto, totalizando, assim, uma amostra de 117 estudantes.

Este estudo foi aprovado pelo Comitê de Ética em Pesquisa da Universidade do Vale do Rio dos Sinos Unisinos (protocolo n. 09/129). Foram seguidos todos os preceitos éticos da Resolução n. 196/96 do Conselho Nacional da Saúde, para pesquisa com seres humanos. Os pais ou responsáveis foram esclarecidos em relação ao Termo de Consentimento Livre e Esclarecido (TCLE) e aos procedimentos adotados na avaliação.

\section{Protocolo de avaliação}

Após a aprovação do projeto pelo Comitê de Ética em Pesquisa, foi encaminhado aos pais ou responsáveis pelas crianças, um questionário fechado, contendo perguntas sobre sinais e sintomas característicos da respiração bucal (18), juntamente com o TCLE, e um convite para sua participação na avaliação.

Em seguida, foi marcado um dia para a avaliação postural, a qual foi realizada por meio de registros fotográficos e análise computadorizada. Após a análise dos questionários e da avaliação postural, foram encaminhados às escolas pesquisadas os resultados encontrados no presente estudo. Cada criança recebeu um documento que continha orientações sobre a respiração bucal, assim como um laudo informando o resultado da sua avaliação postural.

\section{Questionário}

O questionário, adaptado de Abreu et al. (18), foi baseado em critérios clínicos, considerando-se 
respiradoras bucais as crianças que apresentaram dois sinais maiores (ronco; dormir com a boca aberta; baba no travesseiro; queixa de nariz entupido, diariamente) ou um sinal maior, associado a dois ou mais sinais menores (coceira no nariz; queixa de nariz entupido, às vezes; dificuldade para respirar à noite ou sono agitado; sonolência durante o dia; irritabilidade durante o dia; dificuldade ou demora ao engolir alimentos; três ou mais episódios de infecção de garganta ou ouvido, ou sinusite comprovada por médico no último ano; dificuldade no aprendizado escolar).

\section{Avaliação postural}

A avaliação postural foi realizada por meio de análise fotogramétrica, com auxílio do software para Avaliação Postural (SAPO ${ }^{\circledR}$ ), versão 0.67 (19), desenvolvido na Universidade de São Paulo (USP). As imagens foram capturadas com uma câmera fotográfica digital Canon ${ }^{\circledR}$ Power Shot A 620, com 7.1 mega pixels de resolução, apoiada sobre um tripé Goldship ${ }^{\circledR}$ de altura variável.

Para a realização das fotografias, as crianças foram encaminhadas, individualmente, até uma sala previamente preparada para a análise postural. Um fio de prumo foi devidamente afixado ao teto, contendo duas esferas de isopor de 2,5 centímetros de diâmetro, separadas entre si por 50 centímetros e recobertas com papel adesivo reflexivo. A disposição das esferas sobre o fio atuou como marcação para a calibração da imagem no SAPO ${ }^{\circledR}$.

As crianças foram fotografadas vestindo trajes de banho e, quando necessário, com os cabelos presos. Para a marcação dos pontos anatômicos analisados, foram utilizadas esferas de isopor de 2,5 centímetros de diâmetro, recobertas com papel adesivo reflexivo, afixadas com fita dupla face. Constituíram os pontos anatômicos monitorados: trago, acrômio, ângulo inferior da escápula, processo espinhoso T3, espinha ilíaca ântero-superior, espinha ilíaca póstero-superior, ponto sobre a linha média da perna, ponto sobre o tendão calcâneo na altura dos maléolos e calcâneo.

Marcados os pontos, cada criança foi posicionada ao lado do fio de prumo, a uma distância de três metros da câmera, que foi conectada ao tripé, de forma que ficasse a uma altura de cerca da metade da estatura do indivíduo. A avaliação foi constituída por três fotografias: plano sagital direito, plano frontal na vista posterior e plano sagital esquerdo, sempre nessa ordem. Com o objetivo de garantir a mesma base de sustentação nas diferentes vistas, a criança foi orientada a se posicionar, para a primeira tomada de fotografia, sobre um tapete de etilvinilacetato (EVA) preto. Com um giz, então, foi desenhada a marca dos pés nesse tapete. Entre cada tomada das fotografias seguintes, o tapete foi rodado 90 graus, e a criança foi novamente posicionada com os pés apoiados sobre a marca de giz, repetindo, assim, a base de sustentação.

Para a análise da postura, foram selecionadas as seguintes variáveis: (a) Plano frontal, vista posterior: assimetria entre as escápulas e T3, ângulo perna-retropé direito e ângulo perna-retropé esquerdo; (b) Plano sagital: alinhamento vertical da cabeça, alinhamento horizontal da pelve.

\section{Análise estatística}

Foi realizada estatística descritiva pelas medidas de tendência central (média) e de variabilidade (desvio padrão), e a análise da distribuição dos dados foi realizada pelo teste de Kolmogorov-Smirnov. Para a comparação das medidas observadas em relação ao tipo de respirador, utilizou-se o teste t-student $\mathrm{e}$ o teste de Mann-Whitney. A associação foi avaliada pelo teste Exato de Fisher (20).

0 programa Statistical Package to Social Sciences for Windows, versão 17.0 (SPSS - Chicago, IL, USA), foi utilizado em todas as análises, nas quais, para critérios de decisão, foi adotado o nível de significância de $5 \%$.

\section{Resultados}

Os dados serão descritos de acordo com a amostragem total avaliada e, em seguida, será discriminado o resultado por grupo, ou seja, grupo respirador bucal (RB) e grupo respirador nasal (RN).

\section{Caracterização da amostra}

A amostra estudada foi composta por 117 crianças com idade média de 9,017 $\pm 0,851$ anos, sendo $46,15 \%(n=54)$ meninos e $53,85 \%(n=63)$ 
meninas. A estatura média foi de 1,364 $\pm 0,077$ metros e o peso corporal médio foi de $34,943 \pm 9,385$ quilogramas. Em relação ao tipo de respiração, prevaleceram, de forma significativa $\left(\chi_{\text {calc }}^{2}=33,923\right.$; $\mathrm{p}<0,001)$, aquelas com respiração bucal $(76,9 \%$; $=$ 90), quando comparadas às crianças com respiração nasal $(23,1 \%$; $=27)$.

\section{Alinhamento vertical da cabeça}

A Tabela 1 apresenta a distribuição absoluta e relativa para o alinhamento vertical da cabeça, nas vistas lateral direita e esquerda, para a amostra total, bem como para os grupos, RB e RN. Apresenta, ainda, média e desvio padrão em graus. Observa-se o predomínio da anteriorização da cabeça em ambas as vistas, nos dois grupos avaliados. Na comparação dos ângulos observados em cada grupo, nas medidas de anteriorização, nas vistas lateral direita e esquerda, as diferenças observadas não se mostraram expressivas ( $p=0,440$ e $p=0,751$, respectivamente).

Assimetria horizontal das escápulas em relação à $\mathrm{T3}$

Os resultados para assimetria das escápulas em relação à T3 são apresentados na Tabela 2. Verifica-se um predomínio da assimetria à esquerda, que ocorre em ambos os grupos avaliados. Em relação à análise comparativa para assimetria à esquerda, observou-se que as diferenças entre os grupos não se mostraram significativas $(\mathrm{p}=0,231)$.

Tabela 1 - Resultados referentes ao alinhamento vertical da cabeça e à análise estatística

\begin{tabular}{|c|c|c|c|c|c|c|c|}
\hline & \multicolumn{4}{|c|}{ Distribuição absoluta e relativa* } & \multicolumn{3}{|c|}{ Média e desvio padrão (graus) } \\
\hline & Total & RB & RN & $\mathrm{p}$ & RB & RN & $\mathrm{p}$ \\
\hline \multicolumn{8}{|c|}{ Vista Lat Direita } \\
\hline Alinhado & $2(1,7)$ & $1(1,1)$ & $1(3,7)$ & & - & - & - \\
\hline Anteriorização & $89(76,1)$ & $67(74,4)$ & $22(81,5)$ & $0,317^{\natural}$ & $8,1 \pm 5,9$ & $7,0 \pm 4,3$ & $0,440^{\S}$ \\
\hline Posteriorização & $26(22,2)$ & $22(24,4)$ & $4(14,8)$ & & $5,8 \pm 3,6$ & $4,2 \pm 3,3$ & $0,404^{\circ}$ \\
\hline \multicolumn{8}{|c|}{ Vista Lat Esquerda } \\
\hline Alinhado & $9(7,7)$ & $5(5,6)$ & $4(14,8)$ & & - & - & - \\
\hline Anteriorização & $60(51,3)$ & $46(51,1)$ & $14(51,9)$ & $0,278 \pi$ & $7,9 \pm 6,1$ & $7,3 \pm 5,8$ & $0,751^{\S}$ \\
\hline Posteriorização & $48(41,0)$ & $39(43,3)$ & $9(33,3)$ & & $4,8 \pm 3,4$ & $7,4 \pm 3,5$ & $0,051^{\S}$ \\
\hline
\end{tabular}

Fonte: Dados da pesquisa.

Legenda: * $=$ Resultados apresentados da forma $n(\%) ;{ }^{~ "}=$ Teste Exato de Fisher; ${ }^{\Phi}=$ Teste de Mann-Whitney; ${ }^{\S}=$ Teste t-student; $p \leq 0,05$ indica resultado significativo; RB significa respiradores bucais e RN respiradores nasais.

Tabela 2 - Resultados referentes à assimetria horizontal da escápula em relação à T3 e à análise estatística

\begin{tabular}{|c|c|c|c|c|c|c|c|}
\hline & \multicolumn{4}{|c|}{ Distribuição absoluta e relativa* } & \multicolumn{3}{|c|}{ Média e desvio padrão (graus) } \\
\hline & Total & RB & RN & $\mathrm{p}$ & RB & RN & $\mathbf{p}$ \\
\hline Esquerda & $81(69,2)$ & $63(70,0)$ & $18(66,7)$ & & $17,8 \pm 12,7$ & $22,1 \pm 14,4$ & $0,231^{\S}$ \\
\hline Direita & $33(28,2)$ & $24(26,7)$ & $9(33,3)$ & $0,670^{\pi}$ & $13,9 \pm 11,9$ & $25,7 \pm 13,9$ & $0,021^{\Phi}$ \\
\hline Alinhado & $3(2,6)$ & $3(3,3)$ & $0(0,0)$ & & - & - & - \\
\hline
\end{tabular}

Fonte: Dados da pesquisa.

Legenda: * = Resultados apresentados da forma $n(\%) ;{ }^{~ "}=$ Teste Exato de Fisher; ${ }^{\Phi}=$ Teste de Mann-Whitney; ${ }^{\S}=$ Teste t-student; $p \leq 0,05$ indica resultado significativo; RB significa respiradores bucais e RN respiradores nasais. 


\section{Alinhamento horizontal da pelve}

Em relação ao alinhamento horizontal da pelve, predominou a anteroversão em ambas as vistas. Comparando as medidas da anteroversão entre os dois grupos, não foi detectada diferença significativa ( $p=0,971$ na vista lateral direita; $p=0,986$ na vista lateral esquerda). Esses resultados são apresentados na Tabela 3.

\section{Ângulo perna retropé}

A Tabela 4 apresenta os resultados para o ângulo perna/retropé. Pode-se observar a prevalência do calcâneo valgo em ambos os lados, com distribuição semelhante nos dois grupos. Na comparação dos ângulos observados no calcâneo direito e esquerdo, os grupos não apresentaram diferença significativa ( $p=0,936$ e $p=0,404$, respectivamente).

\section{Discussão}

As alterações posturais em crianças e adolescentes são comumente estudadas (21-24). Da mesma forma, são recorrentes as pesquisas acerca da investigação da prevalência de $\mathrm{RB}(2,4,25,26)$ e de alterações posturais em RB $(12,13,27,28)$. Entretanto, não foram encontradas pesquisas sistemáticas acerca da relação desses dois fatores. Sendo assim, o presente estudo teve como objetivo verificar a presença de sinais e sintomas da respiração bucal em uma população, e relacioná-los com as alterações posturais presentes.

Como método de avaliação postural, foi utilizada a análise fotogramétrica, que se apresenta como um método quantitativo confiável $(29,30)$. Para classificar o tipo de respirador, RB ou RN, foi utilizado um questionário adaptado de Abreu et al., que utilizaram um protocolo de avaliação criado pelos próprios autores, visto que não existe na literatura uma ferramenta validada para classificar as crianças RB (18).

Em relação aos sinais e sintomas da respiração bucal, observou-se, neste estudo, que 76,9\% das crianças foram classificadas como RB, resultado similar ao encontrado por Cavassani et al. (31), que verificaram prevalência de 77,78\%. Menezes et al. (25) investigaram a influência de fatores socioeconômicos e demográficos no padrão da respiração, e encontraram, na amostra total, uma prevalência de $55,2 \%$ de crianças RB; porém, quando separadas por escola, pública ou particular, a prevalência subiu para $67,2 \%$ em escola pública, dado mais próximo ao encontrado no presente estudo.

Algumas pesquisas sobre a prevalência de RB apresentam, entretanto, percentuais mais baixos que os verificados no presente estudo. Paula, Leite e Wernek (26) verificaram 59,5\% de RB, dado semelhante aos de Abreu et al. (4), com prevalência de $55 \%$, e Menezes et al. (2), com 53,3\%. Esses estudos não discutem, porém, a influência da classe econômica. Pesquisas indicam que os fatores socioeconômicos influenciam nas condições de saúde. Devido ao difícil

Tabela 3 - Resultados referentes ao alinhamento horizontal da pelve e à análise estatística

\begin{tabular}{|c|c|c|c|c|c|c|c|}
\hline & \multicolumn{4}{|c|}{ Distribuição absoluta e relativa* } & \multicolumn{3}{|c|}{ Média e desvio padrão (graus) } \\
\hline & Total & RB & RN & p & RB & RN & $\mathrm{p}$ \\
\hline \multicolumn{8}{|c|}{ Vista Lat Direita } \\
\hline Anteroversão & $115(98,3)$ & $88(97,8)$ & $27(100,0)$ & \multirow{2}{*}{$1,000^{\pi}$} & $14,9 \pm 5,4$ & $14,8 \pm 4,5$ & $0,971^{\S}$ \\
\hline Retroversão & $2(1,7)$ & $2(2,2)$ & $0(0,0)$ & & $14,6 \pm 17,6$ & - & - \\
\hline \multicolumn{8}{|c|}{ Vista Lat Esquerda } \\
\hline Anteroversão & $116(99,1)$ & $89(98,9)$ & $27(100,0)$ & \multirow{2}{*}{$1,000^{\pi}$} & $13,9 \pm 5,6$ & $13,9 \pm 4,1$ & $0,986^{\S}$ \\
\hline Retroversão & $1(0,9)$ & $1(1,1)$ & $0(0,0)$ & & 3,3 & - & - \\
\hline
\end{tabular}

Fonte: Dados da pesquisa.

Legenda: * $=$ Resultados apresentados da forma n(\%); ${ }^{\circledR}=$ Teste Exato de Fisher; ${ }^{\Phi}=$ Teste de Mann-Whitney; ${ }^{\S}=$ Teste t-student; $p \leq 0,05$ indica resultado significativo; $\mathrm{RB}$ significa respiradores bucais e $\mathrm{RN}$ respiradores nasais. 
Tabela 4 - Resultados referentes ao ângulo perna/retropé e à análise estatística

\begin{tabular}{|c|c|c|c|c|c|c|c|}
\hline & \multicolumn{4}{|c|}{ Distribuição absoluta e relativa* } & \multicolumn{3}{|c|}{ Média e desvio padrão (graus) } \\
\hline & Total & RB & RN & $\mathrm{p}$ & RB & RN & $\mathrm{p}$ \\
\hline \multicolumn{8}{|l|}{ Direito } \\
\hline Calcâneo varo & $6(5,1)$ & $5(5,6)$ & $1(3,7)$ & & $2,1 \pm 1,6$ & 3,1 & - \\
\hline Calcâneo valgo & $111(94,9)$ & $85(94,4)$ & $26(96,3)$ & $1,000^{\pi}$ & $8,3 \pm 5,1$ & $8,4 \pm 4,6$ & $0,936^{\circ}$ \\
\hline Alinhado & $0(0,0)$ & $0(0,0)$ & $0(0,0)$ & & - & - & - \\
\hline \multicolumn{8}{|l|}{ Esquerdo } \\
\hline Calcâneo varo & $18(15,4)$ & $16(17,8)$ & $2(7,4)$ & & $2,8 \pm 2,0$ & $1,5 \pm 0,3$ & $0,377^{\circ}$ \\
\hline Calcâneo valgo & $98(83,8)$ & $73(81,1)$ & $25(92,6)$ & $0,408 \pi$ & $7,8 \pm 4,3$ & $6,8 \pm 3,4$ & $0,404^{\S}$ \\
\hline Alinhado & $1(0,9)$ & $1(1,1)$ & $0(0,0)$ & & - & - & - \\
\hline
\end{tabular}

Fonte: Dados da pesquisa.

Legenda: * = Resultados apresentados da forma n(\%); ${ }^{\pi}=$ Teste Exato de Fisher; ${ }^{\Phi}=$ Teste de Mann-Whitney; ${ }^{\S}=$ Teste t-student; $p \leq 0,05$ indica resultado significativo; RB significa respiradores bucais e RN respiradores nasais.

acesso ao sistema de saúde pública, a população de baixa renda torna-se a de maior risco e, consequentemente, muitos problemas respiratórios acabam não recebendo a devida atenção $(25,31)$. É possível, também, que a variação nos resultados deva-se aos diferentes métodos utilizados, apontando-se como limitação do presente estudo a ausência de associação da avaliação realizada com outros instrumentos para diagnóstico clínico de RB.

Quanto à avaliação postural, verificou-se um elevado número de alterações posturais, visto que todos os alunos apresentaram algum grau de alteração. Santos et al. (15), verificando a ocorrência de desvios posturais em escolares com idades entre 8 e 12 anos, observaram resultado semelhante, tendo em vista que apenas $2 \%$ dos alunos não apresentaram nenhuma alteração postural.

Krakauer e Guilherme (3), ao realizarem avaliação por tipo de respirador, compararam a presença de alterações posturais em crianças RB e RN, de 5 a 10 anos, e verificaram que crianças de 5 a 8 anos, RB ou não, apresentavam alterações posturais. Segundo os autores, isso se justifica pelo fato de que crianças de até 7 ou 8 anos não possuem a musculatura abdominal com função totalmente desenvolvida, e que esse desenvolvimento ocorreria justamente a partir dessa idade. Conforme os pesquisadores, as crianças RN maiores de 8 anos melhoraram a postura, fato não observado nos RB, que continuaram apresentando alterações posturais.
Concordando com o exposto, Yi et al. (8) encontraram diferença significativa entre RB e RN em todas as curvaturas da coluna vertebral e posição da pelve em crianças de 5 a 12 anos. Esses dados discordam do presente estudo, que verificou a presença de alterações posturais em ambos os grupos pesquisados, na faixa etária dos 8 aos 10 anos, assim como Belli et al. (32), que verificaram alterações posturais sem diferença significativa entre RB e RN, na faixa etária dos 7 aos 12 anos.

A ausência de diferença significativa no presente estudo pode estar relacionada ao fato de o questionário ter sido respondido pelos pais, possibilitando a identificação de falsos RN pelo não reconhecimento de características de crianças RB. Logo, a ausência de outros testes para identificação de RB, como o teste de permanência de água na boca $(2,25)$, indica uma limitação desta pesquisa.

No que diz respeito ao posicionamento da cabeça, o presente estudo obteve $76,1 \%$ de crianças com anteriorização da cabeça na vista lateral direita e 51,3\% na esquerda. Apesar de os resultados para as duas vistas serem distintos, a diferença não é significativa $(p=0,603)$. Pode-se explicar a diferença pelo fato de ter sido adotada a sistemática de avaliação da vista lateral direita primeiro, e a esquerda por último, influenciando na mudança da atitude postural. Esses dados são semelhantes aos encontrados por Penha et al. (23), com 58\% na faixa etária de 8 anos, $67 \%$ na de 9 anos, e 64\% na de 10 anos. Percentual mais 
elevado foi encontrado por Detsch e Candotti (22), com $86,21 \%$ na faixa etária dos 10 aos 11 anos; porém, para a faixa etária dos 8 aos 9 anos, esse percentual caiu para $27,27 \%$.

Separando-se por tipo de respirador, este estudo não encontrou diferença significativa, visto que tanto o grupo RB quanto o RN apresentaram anteriorização da cabeça com graus semelhantes nas duas vistas. Verificou-se um percentual de 74,4\% na vista lateral direita e 51,3\% na esquerda no grupo RB e, no grupo RN, $81,5 \%$ na vista direita e $51,9 \%$ na esquerda. Esses resultados discordam dos encontrados por Krakauer e Guilherme (3), que observaram um número menor de alterações no grupo controle em relação ao grupo RB. Pesquisas sobre alterações posturais em $\mathrm{RB}$ apresentam percentuais que compreendem os encontrados no presente estudo, variando de $48,8 \%$ a $89 \%(11,12,33)$. As variações nos resultados das pesquisas podem estar relacionadas ao fato de diferentes métodos e avaliações terem sido utilizados, não existindo um consenso na literatura.

Ao analisar o alinhamento das escápulas, predominou a assimetria à esquerda, que esteve presente em $69,2 \%$ das crianças. Esse predomínio repetiu-se nos grupos, com 70,0\% no RB e $66,7 \%$ no RN, e pode estar relacionado ao lado dominante da criança, visto que, nesta avaliação, a assimetria à esquerda se refere à borda inferior da escápula esquerda mais elevada que a direita, muitas vezes associada a uma depressão do ombro direito; porém, não se pode afirmar, pois a dominância não foi verificada. Não foram encontradas pesquisas com o mesmo método, mas análises que utilizam outras variáveis para caracterizar alterações nas escápulas. Detsch e Candotti (22) realizaram três medidas para alterações nas escápulas e encontraram percentuais variando de $18,18 \%$ a $86,36 \%$ na faixa etária entre 8 e 9 anos, e 41,38\% a $89,66 \%$ na faixa etária dos 10 e 11 anos. Nota-se que o resultado do presente estudo está dentro dessa variação. Yi et al. (12), pesquisando crianças agrupadas de acordo com a etiologia da respiração bucal, observaram percentuais próximos aos encontrados no presente estudo, com prevalência variando de $70 \%$ a $100 \%$.

Não foi encontrada diferença significativa entre os grupos pesquisados, dados que concordam com Krakauer e Guilherme (3), que verificaram assimetria nas escápulas, com valores similares nos grupos controle e RB na faixa etária dos 8 aos 10 anos, sem diferença significativa.
Em relação ao alinhamento da pelve, verificou-se que $98,3 \%$ das crianças apresentaram anteroversão pélvica na vista lateral direita e $99,1 \%$ na esquerda, e esse predomínio ocorreu também nos grupos RB e RN, em ambas as vistas. Em seu estudo, Yi et al. (12) encontraram $81 \%$ de RB com anteroversão pélvica, resultado semelhante ao do presente estudo. Os autores explicam a alta prevalência pelo fato de essa alteração ser fisiológica nessa faixa etária, em decorrência do desenvolvimento da musculatura abdominal a partir dos 8 anos.

Observando o ângulo perna/retropé, 94,9\% das crianças apresentaram calcâneo valgo direito e 83,8\% esquerdo, e esse mesmo predomínio ocorreu em ambos os grupos avaliados. Percentuais inferiores são apresentados por Penha et al. (23), que verificaram uma prevalência de $76 \%$ para crianças de 8 anos e $67 \%$ para crianças de 9 a 10 anos. Não foram encontradas, até o momento, outras pesquisas que utilizassem a medida do calcâneo como variável. 0 calcâneo valgo está relacionado com um aumento de carga na face medial do pé, possibilitando, assim, o surgimento do pé plano. Aos 8 anos, o arco longitudinal medial já está completamente formado, eliminando a possibilidade de uma alteração fisiológica (23).

\section{Conclusão}

Neste estudo foi observado um alto percentual de crianças com sinais e sintomas da respiração bucal, e de alterações posturais. Mesmo sem a constatação da relação da respiração bucal com as alterações posturais, verifica-se que essa população apresenta sérios problemas de saúde pública, os quais podem vir a comprometer a qualidade de vida desses indivíduos.

\section{Referências}

1. Detsch C, Luz AMH, Candotti CT, Oliveira DS, Larazon F, Guimarães LK, et al. Prevalência de alterações posturais em escolares do ensino médio em uma cidade no sul do Brasil. Rev Panam Salud Publica. 2007;21(4): 231-8.

2. Menezes VA, Leal RB, Pessoa RS, Pontes RMES. Prevalência e fatores associados à respiração oral em escolares participantes do projeto Santo Amaro - Recife, 2005. Rev Bras Otorrinolaringol. 2006;72(3):394-9. 
3. Krakauer LH, Guilherme A. Relação entre respiração bucal e alterações posturais em crianças: uma análise descritiva. Rev Dent Press Ortodon Ortopedi Facial. 2000;5(5):85-92.

4. Abreu RR, Rocha RL, Lamounier JA, Guerra AFM. Prevalence of mouth breathing among children. J Pediatr. 2008;84(5):467-70.

5. Costa AVR. Respiração bucal e postura corporal: uma relação de causa e efeito [Internet]. Rio de Janeiro: CEFAC - Saúde e Educação, 1999 [acesso 30 ago. 2009]. Disponível em: http://www.cefac.br/library/teses/8 262012854f96047506de9ff0bfac8aa.pdf/.

6. Paulo CB, Conceição CA. Sintomatologia do respirador oral. Revista CEFAC. 2003;5:219-22.

7. Di Francesco RC, Passerotii G, Paulucci B, Miniti A. Respiração oral na criança: repercussões diferentes de acordo com o diagnóstico. Rev Bras Otorrinolaringol. 2004;70(5):665-70.

8. Yi LC, Jardim JR, Inoue DP, Pignatari SSN. The relationship between excursion of the diaphragm and curvatures of the spiral column in mouth breathing children. J Pediatr (Rio J). 2008;84(2):171-7.

9. Cuccia AM, Lotti M, Caradonna D. Oral breathing and head posture. Angle Orthodontist. 2008;78(1):77-82.

10. Neiva PD, Kirkwood RN, Godinho R. Orientation and position of head posture, scapula and thoracic spine in mouth breathing children. Int J Pediatr Otorhinolaryngol. 2009;73(2):227-36.

11. Falcão DA, Grinfeld S, Grinfeld A, Melo MVR. Respiradores bucais diagnosticados clinicamente e por autodiagnóstico. Conseqüências na postura corporal. Int J Dent. 2003;2(2):250-6.

12. Yi LC, Guedes ZCF, Pignatari S, Weckx LLM. Avaliação postural em crianças de 5 a 12 anos que apresentam respiração oral. Fisioter Mov. 2003;16(3):29-33.

13. Breda D, Moreira HSB. Avaliação postural e da função respiratória em crianças de 5 à 10 anos de idade, com rinite alérgica, hipertrofia de adenóide e síndrome do respirador bucal. Fisioter Brasil. 2003;4(4):247-51.

14. Godoy P, Niitsuma LEM, Caromano FA. Avaliação funcional fisioterapêutica do respirador bucal. Arq Ciênc Saúde da UNIPAR. 2000;4(2):111-20.
15. Santos CIS, Cunha ABN, Braga VP, Saad IAB, Ribeiro MAG, Conti PBM, et al. Ocorrência de desvios posturais em escolares do ensino público fundamental de Jaguariúna, São Paulo. Rev Paul Pediatr. 2009;27(1): 74-80.

16. Corrêa ECR, Bérzin F. Efficacy of physical therapy on cervical muscle activity and on body posture in school-age mouth breathing children. Int J Pediatr Otorhinolaryngol. 2007;71(10):1527-35.

17. Goldim JR. Manual de iniciação à pesquisa em saúde. 2a ed. Porto Alegre: Dacasa; 2000.

18. Abreu RR, Rocha RL, Lamounier JA, Guerra AFM. Etiology, clinical manifestations and concurrent findings in mouth breathing children. J Pediatr. 2008;84(6): 529-35.

19. Portal do projeto software para avaliação postural [Internet]. São Paulo: Incubadora Virtual Fapesp; 2004 [acesso 3 set. 2009]. Disponível em: http://sapo. incubadora.fapesp.br/portal/.

20. Callegari-Jacques SM. Bioestatística: princípios e aplicações. Porto Alegre: Artmed; 2003.

21. Contri DE, Petrucelli A, Perea DCBNM. Incidência de desvios posturais em escolares do $2^{\circ}$ ao $5^{\circ}$ ano do ensino fundamental. ConScientiae Saúde. 2009;8(2):219-24.

22. Detsch C, Candotti CT. A incidência de desvios posturais em meninas de 6 a 17 anos da cidade de Novo Hamburgo. Rev Mov. 2001;7(15):43-56.

23. Penha PJ, João SMA, Casarotto RA, Amino CJ, Penteado DC. Postural assessment of girls between 7 and 10 years of age. Clinics. 2005;60(1):9-16.

24. Penha PJ, Casarotto RA, Sacco ICN, Marques AP, João SMA. Qualitative postural analysis among boys and girls of seven to ten years of age. Rev Bras Fisioter. 2008;12(5):386-91.

25. Menezes VA, Leal RB, Moura MM, Granville-Garcia AF. Influência de fatores socioeconômicos e demográficos no padrão de respiração: um estudo piloto. Rev Bras Otorrinolaringol. 2007;73(6):826-34.

26. Paula MVQ Leite ICG, Wernek RR. Prevalência de portadores da síndrome da respiração bucal na rede escolar do município de Juiz de Fora - MG. HU Rev. 2008;34(1):47-52. 
27. Mancini F, Yi LC, Pisa I, Alves D, Pignatari S. Postural qualitative analysis in mouth breath children. Fisioter Mov. 2007;20(2):119-26.

28. Lima LCO, Baraúna MA, Sologurem MJJ, Canto RST, Gastaldi AC. Postural alterations in children with mouth breathing assessed by computerized biophotogrammetry. J Appl Oral Sci. 2004;12(3):232-7.

29. Santos MM, Silva MPC, Sanada LS, Alves CRJ. Análise postural fotogramétrica de crianças saudáveis de 7 a 10 anos: confiabilidade interexaminadoes. Rev Bras Fisioter. 2009;13(4):350-5.

30. Braz RG, Goes FPDC, Carvalho GA. Confiabilidade e validade de medidas angulares por meio do software para avaliação postural. Fisioter Mov. 2008;21(3):117-26.

31. Cavassani VGS, Ribeiro SG, Nemr NK, Greco AM, Köhle J, Lehn CN. Hábitos orais de sucção: estudo piloto em população de baixa renda. Rev Bras Otorrinolaringol. 2003;69(1):106-10.
32. Belli JFC, Chaves TC, Oliveira AS, Grossi DB. Analysis of body in children with mild to moderate asthma. Eur J Pediatr. 2009;168(10):1207-16.

33. Basso DBA, Souza JA, Pasinato F, Corrêa ECR, Silva AMT. Estudo da postura corporal em crianças com respiração predominantemente oral e escolares em geral. Saúde. 2009;35(1):21-7.

Recebido: 07/ 12/2010

Received: $12 / 07 / 2010$

Aprovado: 01/08/2011

Approved: 08/01/2011 